\title{
Relativistic Energy Analysis of Five-Dimensional q-Deformed Radial Rosen-Morse Potential Combined with q-Deformed Trigonometric Scarf Noncentral Potential Using Asymptotic Iteration Method
}

\author{
Subur Pramono, ${ }^{1}$ A. Suparmi, ${ }^{2}$ and Cari Cari ${ }^{2}$ \\ ${ }^{1}$ Physics Department, Graduate Program, Sebelas Maret University, Jl. Ir. Sutami 36A Kentingan, Surakarta 57126, Indonesia \\ ${ }^{2}$ Physics Department, Faculty of Mathematics and Fundamental Science, Sebelas Maret University, Jl. Ir. Sutami 36A Kentingan, \\ Surakarta 57126, Indonesia \\ Correspondence should be addressed to Subur Pramono; suburpramono.26@gmail.com
}

Received 28 March 2016; Revised 20 July 2016; Accepted 31 July 2016

Academic Editor: Edward Sarkisyan-Grinbaum

Copyright (C) 2016 Subur Pramono et al. This is an open access article distributed under the Creative Commons Attribution License, which permits unrestricted use, distribution, and reproduction in any medium, provided the original work is properly cited. The publication of this article was funded by SCOAP S $^{3}$

\begin{abstract}
We study the exact solution of Dirac equation in the hyperspherical coordinate under influence of separable $q$-deformed quantum potentials. The $q$-deformed hyperbolic Rosen-Morse potential is perturbed by $q$-deformed noncentral trigonometric Scarf potentials, where all of them can be solved by using Asymptotic Iteration Method (AIM). This work is limited to spin symmetry case. The relativistic energy equation and orbital quantum number equation $l_{D-1}$ have been obtained using Asymptotic Iteration Method. The upper radial wave function equations and angular wave function equations are also obtained by using this method. The relativistic energy levels are numerically calculated using Matlab, and the increase of radial quantum number $n$ causes the increase of bound state relativistic energy level in both dimensions $D=5$ and $D=3$. The bound state relativistic energy level decreases with increasing of both deformation parameter $q$ and orbital quantum number $n_{l}$.
\end{abstract}

\section{Introduction}

Dirac equation as relativistic wave equation was formulated by P. A. M Dirac in 1928; the exact solution of Dirac equation for some quantum potentials plays a fundamental role in relativistic quantum mechanics [1]. In order to investigate nuclear shell model, spin symmetry and pseudospin symmetry solutions of Dirac equations have been an important field of study in nuclear physics. The concept of spin symmetry and pseudospin symmetry limit with nuclear shell model has been used widely in explaining a number of phenomena in nuclear physics and related field [2]. In nuclear physics, spin symmetry and pseudospin symmetry concepts have been used to study the aspect of deformed and super deformation nuclei. The concept of spin symmetry has been applied to the level of meson and antinucleon [3]. Pseudospin symmetry has been observed in deformed nuclei and can be enhanced in heavy proton-rich nuclei [4].

Solutions of Dirac equation for some potentials under limit case of spin symmetry and pseudospin symmetry have been investigated intensively whether in three- $[5,6]$, two-, or one- [7-13] dimensional space some $D$-dimensional spherical symmetric spacetimes $[14,15]$. However, The $D$-dimensional Dirac equation with $(D-1)$-dimensional separable noncentral potential has not been investigated yet; therefore, it may be worthy to investigate Dirac equation in 5 dimensions with separable 4-dimensional noncentral potential in this study.

In recent years, some researchers have studied solution of Dirac equation with quantum potentials with different application and methods. These investigations include Eckart potential and trigonometric Manning-Rosen potential 
using Asymptotic Iteration Method (AIM) [5], q-deformed hyperbolic Pöschl-Teller potential and trigonometric Scarf II noncentral potential using Nikiforov-Uvarov method [6], $q$-deformed trigonometric Scarf potential with $q$-deformed Trigonometric Tensor Coupling Potential for Spin and Pseudospin Symmetries Using Romanovski Polynomial [7], generalized nuclear Wood-Saxon potential under relativistic spin symmetry limit [8], relativistic bound states of particle in Yukawa field with Coulomb tensor interaction [9], RosenMorse potential including the spin-orbit centrifugal term using Nikiforov-Uvarov (NU) method [3], pseudospin symmetric solution of the Morse potential for any $\kappa$ state using AIM [11], Scalar, Vector, and Tensor Cornell Interaction using Ansatz method [12], Scalar and Vector Generalized Isotonic Oscillators and Cornell Tensor Interaction using Ansatz method [13], Mie-type potentials for energy dependent pseudoharmonic potential via SUSYQM [16], trigonometric Scarf potential in $D$-dimension for spin and pseudospin symmetry using Nikiforov-Uvarov (NU) method [15], Coulombic potential and its thermodynamics properties in $D$-dimensional space using NU method [17], and hyperbolic tangent potential and its application in material properties in $D$-dimensional space [18].

Asymptotic Iteration Methods (AIM) have small deviation for determination of eigenenergies and eigenfunctions of Dirac equation. The separable $D$-dimensional quantum potentials are not studied yet by some researchers. In this paper, we use Asymptotic Iteration Method (AIM) to solve the Dirac equation under influence of separable $D$-dimensional quantum potentials. The relativistic energy levels can be obtained from calculation of relativistic energy equation using Matlab R2013a. In Section 2, we present basic theory of Dirac equation in hyperspherical coordinate with $D$ dimensional separable quantum potential. In this section, deformed quantum potential is also included which is proposed by Dutra in 2005 [19]. In Section 3, we present Asymptotic Iteration Method. Result and discussion are included in Section 4, and in Section 5 we present the special case in 3dimensional space. In the last section, we present conclusion.

\section{Dirac Equation with Separable q-Deformed Quantum Potential in the Hyperspherical Coordinates}

For single particle, Dirac equation with vector potential $V(r)$ and scalar potential $S(r)$ in the hyperspherical coordinate can be expressed as follows (in the unit $\hbar=c=1$ ) $[13,14]$ :

$$
\begin{aligned}
\{\widehat{\alpha} \cdot \vec{p}+\widehat{\beta}(M+S(\vec{r}))\} \psi(\vec{r}) \\
\quad=\{E-V(\vec{r})\} \psi(\vec{r})
\end{aligned}
$$

where $\vec{p}, E$, and $M$ are $D$-dimensional momentum operator, total relativistic energy, and relativistic mass of the particle, respectively:

$$
\widehat{\alpha}_{i}=\left(\begin{array}{cc}
0 & \widehat{\sigma}_{i} \\
\widehat{\sigma}_{i} & 0
\end{array}\right),
$$

$$
\widehat{\beta}=\left(\begin{array}{cc}
1 & 0 \\
0 & -1
\end{array}\right),
$$

where $\widehat{\sigma}_{i}$ are Pauli's matrices and $\mathbf{1}$ is the $2 \times 2$ unit matrix. Here we use relations between Pauli's matrices as

$$
\widehat{\sigma}_{i} \widehat{\sigma}_{j}+\widehat{\sigma}_{j} \widehat{\sigma}_{i}=2 \delta_{i j} \mathbf{1}
$$

The wave function of Dirac spinor can be classified in two forms, upper spinor $\chi(\vec{r})$ and lower spinor $\varphi(\vec{r})$ as follows $[15,16]$ :

$$
\begin{aligned}
& \psi(\vec{r})=\left(\begin{array}{l}
\chi(\vec{r}) \\
\varphi(\vec{r})
\end{array}\right) \\
& =\left(\begin{array}{l}
\frac{F_{n k}(r)}{r^{(D-1) / 2}} Y_{\ell_{1}, \ldots, \ell_{D-1}}^{\ell}\left(\hat{x}=\theta_{1}, \theta_{2}, \ldots, \theta_{D-1}\right) \\
i \frac{G_{n k}(r)}{r^{(D-1) / 2}} Y_{\tilde{\ell}_{1}, \ldots, \tilde{\ell}_{D-1}}^{\tilde{\ell}}\left(\hat{x}=\theta_{1}, \theta_{2}, \ldots, \theta_{D-1}\right)
\end{array}\right) .
\end{aligned}
$$

By substituting (2), (3), and (5) into (1), we get

$$
\begin{aligned}
& \vec{\sigma} \cdot \vec{p} \varphi(\vec{r})=-(M-E+(V(\vec{r})+S(\vec{r}))) \chi(\vec{r}), \\
& \vec{\sigma} \cdot \vec{p} \chi(\vec{r})=(M+E-(V(\vec{r})-S(\vec{r}))) \varphi(\vec{r}) .
\end{aligned}
$$

Exact spin symmetry limit is characterized with $V(\vec{r})=$ $S(r)$, but for spin symmetry limit $V(r)-S(r)=\Delta$ which is constant, and for pseudospin symmetry limit $V(r)+S(r)=$ $\Sigma$ which is also constant. For spin symmetry limit we have $d \Delta(\vec{r}) / d r=0$ since $\Delta(\vec{r})=C_{s}=$ constant; therefore, (6) can be rewritten as

$$
\begin{aligned}
\vec{\sigma} \cdot \vec{p} \varphi(\vec{r}) & =-\{(M-E)+\Sigma(\vec{r})\} \chi(\vec{r}), \\
\varphi(\vec{r}) & =\frac{\vec{\sigma} \cdot \vec{p}}{\left(M+E-C_{s}\right)} \chi(\vec{r}) .
\end{aligned}
$$

If (8) is substituted into (7) we get

$$
\begin{aligned}
& \vec{p}^{2} \chi(\vec{r}) \\
& \quad=\left\{(M+E)-C_{s}\right\}[-\{(M-E)+(\Sigma(\vec{r}))\}] \chi(\vec{r}),
\end{aligned}
$$

where

$$
(\vec{\sigma} \cdot \vec{p})(\vec{\sigma} \cdot \vec{p})=\vec{p}^{2}
$$

Using momentum operator definition in quantum mechanics, where $\vec{p}=-i \vec{\nabla}_{D}$, the hyperspherical Laplacian $\vec{\nabla}_{D}^{2}$ is given as [15]

$$
\nabla_{D}^{2}=\frac{1}{r^{D-1}} \frac{\partial}{\partial r}\left(r^{D-1} \frac{\partial}{\partial r}\right)+\left(\frac{L_{D-1}^{2}}{r^{2}}\right) .
$$

The eigenvalue of $L_{D-1}^{2}$ is $\ell_{D-1}^{\prime}\left(\ell_{D-1}^{\prime}+D-2\right)$ and the angular momentum operator is expressed as [15]

$$
\begin{aligned}
L_{D-1}^{2} & =-\left\{\frac{1}{\sin ^{D-2} \theta_{D-1}} \frac{\partial}{\partial \theta_{D-1}}\left(\sin ^{D-2} \theta_{D-1} \frac{\partial}{\partial \theta_{D-1}}\right)\right. \\
& \left.-\frac{L_{D-2}^{2}}{\sin ^{2} \theta_{D-1}}\right\} .
\end{aligned}
$$


By inserting (11) into (9), we get

$$
\begin{aligned}
\frac{1}{r^{D-1}} & \frac{\partial}{\partial r}\left(r^{D-1} \frac{\partial}{\partial r} \chi(\vec{r})\right)+\left(\frac{L_{D-1}^{2}}{r^{2}}\right) \chi(\vec{r}) \\
& -\left(M^{2}-E^{2}\right) \chi(\vec{r})-\left\{(M+E)-C_{s}\right\} \sum(\vec{r}) \chi(\vec{r}) \\
\quad= & 0 .
\end{aligned}
$$

The separable variable potential used in this study is $q$-deformed hyperbolic Rosen-Morse potential plus qdeformed noncentral Scarf trigonometric potential in hyperspherical coordinate space. The effective potential can be written as

$$
\begin{aligned}
& \Sigma(\bar{r})=\left[\left\{-\frac{V_{0}}{\cosh _{q}^{2}(\alpha r)}+V_{1} \tanh _{q}(\alpha r)\right\}\right. \\
& +\frac{1}{r^{2}}\left\{\frac{V\left(\theta_{1}\right)}{\sin ^{2} \theta_{2} \sin ^{2} \theta_{3} \sin ^{2} \theta_{4}}+\frac{V\left(\theta_{2}\right)}{\sin ^{2} \theta_{3} \sin ^{2} \theta_{4}}\right. \\
& \left.\left.+\frac{V\left(\theta_{3}\right)}{\sin ^{2} \theta_{4}}+V\left(\theta_{4}\right)\right\}\right]
\end{aligned}
$$

with angular potentials $V\left(\theta_{i}\right)$ taken as

$$
\begin{aligned}
& V\left(\theta_{1}\right)=\frac{b_{1}^{2}+a_{1}\left(a_{1}-1\right)}{\sin _{q}^{2} \theta_{1}}-\frac{2 b_{1}\left(a_{1}-1 / 2\right) \cos _{q} \theta_{1}}{\sin _{q}^{2} \theta_{1}}, \\
& V\left(\theta_{2}\right)=\frac{b_{2}^{2}+a_{2}\left(a_{2}-1\right)}{\sin _{q}^{2} \theta_{2}}-\frac{2 b_{2}\left(a_{2}-1 / 2\right) \cos _{q} \theta_{2}}{\sin _{q}^{2} \theta_{2}}, \\
& V\left(\theta_{3}\right)=\frac{b_{3}^{2}+a_{3}\left(a_{3}-1\right)}{\sin _{q}^{2} \theta_{3}}-\frac{2 b_{3}\left(a_{3}-1 / 2\right) \cos _{q} \theta_{3}}{\sin _{q}^{2} \theta_{3}}, \\
& V\left(\theta_{4}\right)=\frac{b_{4}{ }^{2}+a_{4}\left(a_{4}-1\right)}{\sin _{q}^{2} \theta_{4}}-\frac{2 b_{4}\left(a_{4}-1 / 2\right) \cos _{q} \theta_{4}}{\sin _{q}^{2} \theta_{4}} .
\end{aligned}
$$

By substituting (14) into (13) and using variable separation method, we get the radial part and the angular part of Dirac equations in hyperspherical coordinate with $D=5$.

2.1. The Radial Part. The D-dimensional Dirac equation with $q$-deformed hyperbolic Rosen-Morse potential plus $q$ deformed trigonometric Scarf noncentral potentials can be resolved into the form of radial part and angular part equations. The radial part of $D$-dimensional Dirac equation in this case can be expressed as

$$
\begin{aligned}
& \frac{d^{2} F_{n k}(r)}{d r^{2}}-\frac{\left(\ell_{D-1}^{\prime}+(D-1) / 2\right)\left(\ell_{D-1}^{\prime}+(D-3) / 2\right)}{r^{2}} \\
& \cdot F_{n k}(r) \\
& +\left[\left\{\frac{V_{0}}{\cosh _{q}^{2}(\alpha r)}-V_{1} \tanh _{q}(\alpha r)\right\}\{M+E\}\right. \\
& \left.-\left\{M+E-C_{s}\right\}\{M-E\}\right] F_{n k}(r)=0
\end{aligned}
$$

with $\lambda_{4}=\lambda_{D-1}=\ell_{D-1}^{\prime}\left(\ell_{D-1}^{\prime}+D-2\right)$.
2.2. The Angular Part. The angular part of 5-dimensional Dirac equation obtained from (13)-(14) can be resolved into four parts, and for $C_{s}=0$, we get

$$
\begin{aligned}
& \frac{1}{P_{1}\left(\theta_{1}\right)}\left(\frac{\partial^{2} P_{1}\left(\theta_{1}\right)}{\partial \theta_{1}^{2}}\right)+\left\{\lambda_{1}-(M+E) V\left(\theta_{1}\right)\right\}=0 \\
& \frac{1}{P_{2}\left(\theta_{2}\right)}\left\{\frac{1}{\sin \theta_{2}} \frac{\partial}{\partial \theta_{2}}\left(\sin \theta_{2} \frac{\partial P_{2}\left(\theta_{2}\right)}{\partial \theta_{2}}\right)\right\} \\
& \quad+\left\{\lambda_{2}-\frac{\lambda_{1}}{\sin ^{2} \theta_{2}}-(M+E) V\left(\theta_{2}\right)\right\}=0 \\
& \frac{1}{P_{3}\left(\theta_{3}\right)} \frac{1}{\sin ^{2} \theta_{3}} \frac{\partial}{\partial \theta_{3}}\left(\sin ^{2} \theta_{3} \frac{\partial P_{3}\left(\theta_{3}\right)}{\partial \theta_{3}}\right) \\
& +\left\{\lambda_{3}-\frac{\lambda_{2}}{\sin ^{2} \theta_{3}}-(M+E) V\left(\theta_{3}\right)\right\}=0 \\
& \frac{1}{P_{4}\left(\theta_{4}\right)} \frac{1}{\sin ^{3} \theta_{4}} \frac{\partial}{\partial \theta_{4}}\left(\sin ^{3} \theta_{4} \frac{\partial P_{4}\left(\theta_{4}\right)}{\partial \theta_{4}}\right) \\
& \quad+\left\{\lambda_{4}-\frac{\lambda_{3}}{\sin ^{2} \theta_{4}}-\{M+E\} V\left(\theta_{4}\right)\right\}=0 .
\end{aligned}
$$

Equations (20)-(23) are the angular part of Dirac equation for $\theta_{1}$ until $\theta_{4}$, respectively. The $D$-dimensional relativistic wave functions and orbital quantum numbers are obtained from those equations.

\section{Review of Asymptotic Iteration Method (AIM)}

Asymptotic Iteration Method (AIM) is an alternative method which has accuracy and high efficiency to determine eigenenergies and eigenfunctions for analytically solvable hyperbolic-like potential. Asymptotic Iteration Method is also giving solution for exactly solvable problem [17].

AIM is used to solve the second-order homogeneous linear equation as follows $[5,20-23]$ :

$$
y_{n}^{\prime \prime}(x)=\lambda_{0}(x) y_{n}^{\prime}(x)+S_{0}(x) y_{n}(x),
$$

where $\lambda_{0}(x) \neq 0$ and prime symbol refers to derivation along $x$. The other parameter $n$ is interpreted as radial quantum number. Variables $\lambda_{0}(x)$ and $S_{0}(x)$ are variables that can be differentiated along $x$. To get the solution of (24), we have to differentiate (24) along $x$, and then we get

$$
y_{n}^{\prime \prime \prime}(x)=\lambda_{1}(x) y_{n}^{\prime}(x)+S_{1}(x) y_{n}(x)
$$

where

$$
\begin{aligned}
& \lambda_{1}(x)=\lambda_{0}^{\prime}(x)+S_{0}(x)+\lambda_{0}^{2}(x), \\
& S_{1}(x)=S_{0}^{\prime}(x)+S_{0}(x) \lambda_{0}(x) .
\end{aligned}
$$

Asymptotic Iteration Method (AIM) can be applied exactly in the different problem if the wave function has been known and fulfills boundary conditions zero (0) and infinity $(\infty)$. 
Equation (2) can be simply iterated until $(k+1)$ and $(k+2)$, $k=1,2,3, \ldots$ and then we get

$$
\begin{aligned}
y_{n}{ }^{k+1}(x) & =\lambda_{k-1}(x) y_{n}^{\prime}(x)+S_{k-1}(x) y_{n}(x), \\
y_{n}{ }^{k+2}(x) & =\lambda_{k}(x) y_{n}^{\prime}(x)+S_{k}(x) y_{n}(x), \\
\lambda_{k}(x) & =\lambda_{k-1}^{\prime}(x)+S_{k-1}(x)+\lambda_{0}(x) \lambda_{k-1}(x), \\
S_{k}(x) & =S_{k-1}^{\prime}(x)+S_{0}(x) \lambda_{k-1}(x)
\end{aligned}
$$

which is called as recurrence relation. Eigenvalue can be found using equation given as

$$
\Delta_{k}(z)=\lambda_{k}(z) s_{k-1}(z)-\lambda_{k-1}(z) s_{k}(z)=0,
$$

where $k=1,2,3, \ldots$ is the iteration number and $n_{r}$ is representation of radial quantum number.

Equation (2) is the second-order homogeneous linear equation which can be solved by comparing it with the second-order linear equation as follows [20]:

$$
\begin{aligned}
y^{\prime \prime}(z)= & 2\left(\frac{a z^{N+1}}{1-b z^{N+2}}-\frac{t+1}{z}\right) y^{\prime}(z) \\
& -\frac{w z^{N}}{1-b z^{N+2}} y(z),
\end{aligned}
$$

where

$$
\begin{aligned}
(\sigma)_{n} & =\frac{\Gamma(\sigma+n)}{\Gamma(\sigma)}, \\
\sigma & =\frac{2 t+N+3}{N+2}, \\
\rho & =\frac{(2 t+1) b+2 a}{(N+2) b} .
\end{aligned}
$$

The wave function of (2) is the solution of (32) which is given as [20]

$$
\begin{aligned}
& y_{n}(z) \\
& \quad=(-1)^{n} C_{2}(N+2)^{n}(\sigma)_{n 2} F_{1}\left(-n, \rho+n ; \sigma ; b z^{N+2}\right) .
\end{aligned}
$$

The $q$-deformed hyperbolic and trigonometric functions are used as one of the parameters in the modified RosenMorse potential and noncentral Scarf trigonometric potentials were defined by Arai [24] some years ago as follows:

$$
\begin{aligned}
\sinh _{q} \alpha r & =\frac{e^{\alpha r}-q e^{-\alpha r}}{2}, \\
\cosh _{q} \alpha r & =\frac{e^{\alpha r}+q e^{-\alpha r}}{2}, \\
\tanh _{q} \alpha r & =\frac{\sinh _{q} \alpha r}{\cosh _{q} \alpha r}, \\
\cosh _{q}^{2} \alpha r-\sinh _{q}^{2} \alpha r & =q .
\end{aligned}
$$

Deformation with $q$-parameter in the hyperbolic function can be extended into trigonometric function. Definition of trigonometric function can be arranged by the same way as in the hyperbolic function introduced by Suparmi et al. [7] as follows:

$$
\begin{aligned}
\sin _{q} a r & =\frac{e^{i a r}-q e^{-i a r}}{2}, \\
\cos _{q} a r & =\frac{e^{i a r}+q e^{-i a r}}{2}, \\
\cos _{q}^{2} a r+\sin _{q}^{2} a r & =q, \\
\tan _{q} a r & =\frac{\sin _{q} a r}{\cos _{q} a r}, \\
\sec _{q} a r & =\frac{1}{\cos _{q} a r}, \\
\frac{d \sin _{q} a r}{d r} & =a \cos _{q} a r, \\
\frac{d \cos _{q} a r}{d r} & =-a \sin _{q} a r, \\
\frac{d \tan _{q} a r}{d r} & =q a \sec _{q}^{2} a r .
\end{aligned}
$$

By a convenient translation of spatial variable, one can transform the deformed potentials into the form of nondeformed potentials or vice-versa. In analogy to the translation of spatial variable for hyperbolic function introduced by Dutra [19], we propose the translation of spatial variable for hyperbolic and trigonometric function as follows:

$$
\begin{aligned}
& r \longrightarrow r+\frac{\ln \sqrt{q}}{\alpha}, \\
& r \longrightarrow r-\frac{\ln \sqrt{q}}{\alpha}, \\
& r \longrightarrow r+\frac{\ln \sqrt{q}}{i \alpha}, \\
& r \longrightarrow r-\frac{\ln \sqrt{q}}{i \alpha} .
\end{aligned}
$$

And then by inserting (21) into (18) and (19), we have

$$
\begin{gathered}
\sinh _{q} \alpha r \longrightarrow \sqrt{q} \sinh \alpha r ; \\
\cosh _{q} \alpha r \longrightarrow \sqrt{q} \cosh \alpha r ; \\
\sin _{q} \alpha r \longrightarrow \sqrt{q} \sin \alpha r ; \\
\cos _{q} \alpha r \longrightarrow \sqrt{q} \cos \alpha r
\end{gathered}
$$

The translation of spatial variable in (38) can be used to map the energy and wave function of nondeformed potential toward deformed potential of Scarf potential. 


\section{Result and Discussion}

4.1. Radial Part. The radial part of Dirac equation in hyperspherical space when $C_{s}=0$ can be expressed as

$$
\begin{aligned}
& \frac{d^{2} F_{n k}(r)}{d r^{2}}-\frac{\left(\ell_{D-1}^{\prime}+(D-1) / 2\right)\left(\ell_{D-1}^{\prime}+(D-3) / 2\right)}{r^{2}} \\
& \cdot F_{n k}(r) \\
& +\left[\left\{\frac{V_{0}}{\cosh _{q}^{2}(\alpha r)}-V_{1} \tanh _{q}(\alpha r)\right\}\{M+E\}\right. \\
& \left.-\left\{M^{2}-E^{2}\right\}\right] F_{n k}(r)=0 .
\end{aligned}
$$

Equation (39) cannot be solved directly when $\ell_{D-1}^{\prime} \neq 0$; in this condition, we use approximation to solve centrifugal term with Pekeris approximation. Because we have used $q$ deformed quantum potential, the Pekeris approximation in this condition can be expressed as [3]

$$
\frac{1}{r^{2}}=\frac{1}{r_{e}^{2}}\left(c_{0}+c_{1} \frac{-e^{-2 \alpha r}}{1+q e^{-2 \alpha r}}+c_{2}\left(\frac{-e^{-2 \alpha r}}{1+q e^{-2 \alpha r}}\right)^{2}\right)
$$

with

$$
\begin{aligned}
c_{0}= & 1-\left(\frac{1+q \exp \left(-2 \alpha r_{e}\right)}{2 \alpha r_{e}}\right)^{2}\left(\frac{8 \alpha r_{e}}{1+q \exp \left(-2 \alpha r_{e}\right)}\right. \\
& \left.-\left(3+2 \alpha r_{e}\right)\right), \\
c_{1} & =-2\left(\exp \left(2 \alpha r_{e}\right)+1\right)\left(3\left(\frac{1+q \exp \left(-2 \alpha r_{e}\right)}{2 \alpha r_{e}}\right)\right. \\
& \left.-\left(3+2 \alpha r_{e}\right)\left(\frac{1+q \exp \left(-2 \alpha r_{e}\right)}{2 \alpha r_{e}}\right)\right), \\
c_{2} & =\left(\exp \left(2 \alpha r_{e}\right)+1\right)^{2}\left(\frac{1+q \exp \left(-2 \alpha r_{e}\right)}{2 \alpha r_{e}}\right)^{2}(3 \\
& \left.+2 \alpha r_{e}-\frac{4 \alpha r_{e}}{1+q \exp \left(-2 \alpha r_{e}\right)}\right) .
\end{aligned}
$$

By inserting (38) into (37a) and (37b) and changing from exponential form to hyperbolic form, we get

$$
\begin{aligned}
& \frac{d^{2} F_{n k}(r)}{d r^{2}} \\
& -\left(\omega\left(c_{0}-\frac{c_{1}}{2 \sqrt{q}}+\frac{c_{2}}{2 q}\right)+\left\{M^{2}-E^{2}\right\}\right) F_{n k}(r) \\
& -\left\{\omega\left(\frac{c_{1}}{2 \sqrt{q}}-\frac{c_{2}}{2 q}\right)+V_{1}\{M+E\}\right\} \tanh _{q} \alpha r F_{n k}(r) \\
& +\left[\left\{\frac{\omega c_{2}}{4}+V_{0}\{M+E\}\right\} \frac{1}{\cosh _{q}^{2}(\alpha r)}\right] F_{n k}(r)=0
\end{aligned}
$$

with

$$
\frac{\left(\ell_{D-1}^{\prime}+(D-1) / 2\right)\left(\ell_{D-1}^{\prime}+(D-3) / 2\right)}{r_{e}^{2}}=\omega,
$$

where $r_{e}$ is equilibrium distance that can be derived by potential parameter.

Let us assume

$$
\begin{aligned}
& \alpha^{2} E^{\prime}=-\left(\omega\left(c_{0}-\frac{c_{1}}{2 \sqrt{q}}+\frac{c_{2}}{2 q}\right)+\left\{M^{2}-E^{2}\right\}\right), \\
& \alpha^{2} \rho=\left\{\omega\left(\frac{c_{1}}{2 \sqrt{q}}+\frac{c_{2}}{2 q}\right)+V_{1}\{M+E\}\right\}, \\
& \alpha^{2} \nu(\nu+1)=\left\{\frac{\omega c_{2}}{4}+V_{0}\{M+E\}\right\} .
\end{aligned}
$$

Equations (44) are inserted into (40) so we obtain

$$
\begin{aligned}
& \frac{d^{2} F_{n k}(r)}{d r^{2}} \\
& \quad-\alpha^{2}\left\{\rho \tanh _{q} \alpha r-\nu(\nu+1) \operatorname{sech}_{q}^{2}(\alpha r)\right\} F_{n k}(r) \\
& =-\alpha^{2} E^{\prime} F_{n k}(r) .
\end{aligned}
$$

Let

$$
\tanh _{q} \alpha r=1-2 z .
$$

By using (45)-(46), we get

$$
\begin{aligned}
z(1-z) & \frac{\partial^{2} F_{n k}(z)}{\partial z^{2}}+(1-2 z) \frac{\partial F_{n k}(z)}{\partial z} \\
& -\frac{\left\{\rho \tanh _{q} \alpha r-\nu(\nu+1) \operatorname{sech}_{q}^{2} \alpha r\right\}}{4 z(1-z)} F_{n k}(z) \\
= & -\frac{E^{\prime}}{4 z(1-z)} F_{n k}(z) .
\end{aligned}
$$

By substituting (46) and $\operatorname{sech}_{q}^{2} \alpha r=4 z(1-z) / q$ into (47), we get

$$
\begin{aligned}
& z(1-z) \frac{\partial^{2} F_{n k}(z)}{\partial z^{2}}+(1-2 z) \frac{\partial F_{n k}(z)}{\partial z} \\
& \quad+\left\{\frac{\nu(\nu+1)}{q}-\left(\frac{\rho-E^{\prime}}{4 z}\right)-\left(\frac{-\rho-E^{\prime}}{4(1-z)}\right)\right\} F_{n k}(z) \\
& \quad=0 .
\end{aligned}
$$

Equation (48) has two regular singular points for $z=0$ and $z=1$ so the general solution from (48) is $F_{n \kappa}(z)=z^{\delta}(1-$ $z)^{\gamma} f_{n}(z)$.

Let $4 \delta^{2}=\rho-E^{\prime}$ and $4 \gamma^{2}=-\rho-E^{\prime}$ and then substitute them into (48) to get

$$
\begin{aligned}
& z(1-z) f_{n}^{\prime \prime}(z)+\{(2 \delta+1)-z(2 \delta+2 \gamma+2)\} f_{n}^{\prime}(z) \\
& +\left\{\frac{\nu(\nu+1)}{q}-(\delta+\gamma)(\delta+\gamma+1)\right\} f_{n}(z)=0 .
\end{aligned}
$$


Equation (49) is the second differential equation that can be manipulated into the form as in (22):

$$
\begin{aligned}
& f_{n}^{\prime \prime}(z) \\
& =\frac{\{z(2 \delta+2 \gamma+2)\}-(2 \delta+1)}{z(1-z)} f_{n}^{\prime}(z) \\
& \quad+\frac{\{(\delta+\gamma)(\delta+\gamma+1)-\nu(\nu+1) / q\}}{z(1-z)} f_{n}(z), \\
& \lambda_{0}(z)=\frac{z(2 \delta+2 \gamma+2)-(2 \delta+1)}{z(1-z)}, \\
& S_{0}(z)=\frac{\{(\delta+\gamma)(\delta+\gamma+1)-v(\nu+1) / q\}}{z(1-z)} .
\end{aligned}
$$

Let $A=(\delta+\gamma)(\delta+\gamma+1)-\nu(\nu+1) / q$ and using (29)-(30), together with (51) and (52), we get

$$
\begin{aligned}
& \lambda_{1}(z) \\
& =\left\{\frac{(2 \delta+1)}{z^{2}}+\frac{(2 \gamma+1)}{(1-z)^{2}}\right\}+\left\{\frac{A}{z}+\frac{A}{(1-z)}\right\} \\
& +\left\{-\frac{(2 \delta+1)}{z}+\frac{(2 \gamma+1)}{(1-z)}\right\}^{2}, \\
& =\left\{-\frac{A}{z^{2}}+\frac{A}{(1-z)^{2}}\right\} \\
& +\left\{\frac{A}{z}+\frac{A}{(1-z)}\right\}\left\{-\frac{(2 \delta+1)}{z}+\frac{(2 \gamma+1)}{(1-z)}\right\} .
\end{aligned}
$$

$$
\begin{gathered}
\Delta_{1}=s_{0} \lambda_{1}-s_{1} \lambda_{0}=0 \longrightarrow \\
\varepsilon_{0}=(\delta+\gamma)(\delta+\gamma+1) \\
\Delta_{2}=s_{1} \lambda_{2}-s_{2} \lambda_{2}=0 \longrightarrow \\
\varepsilon_{1}=(\delta+\gamma+1)(\delta+\gamma+2) \\
\vdots \\
\varepsilon_{n}=(\delta+\gamma+n)(\delta+\gamma+n+1),
\end{gathered}
$$

where $\varepsilon_{n}$ is $n$th eigenvalue when $n=0,1,2, \ldots$ and if $n$ is radial quantum number. By using (29)-(30) and the last equation in (55), we have

$$
\begin{aligned}
\varepsilon_{n} & =\frac{\nu(\nu+1)}{q} \\
& =\left\{\frac{\left(\ell_{4}^{\prime}+3 / 2\right)\left(\ell_{4}^{\prime}+1 / 2\right)}{r_{e}^{2} 4 \alpha^{2} q} c_{2}+\frac{V_{0}(M+E)}{\alpha^{2} q}\right\} .
\end{aligned}
$$

The last equation in (55) can be rewritten as

$$
\varepsilon_{n}=(\delta+\gamma+(n+1))^{2}-(\delta+\gamma+(n+1)) .
$$

From (57), we get that the relativistic energy equation of this system is

$$
\begin{aligned}
& \left(M^{2}-E^{2}\right)=\alpha^{2}\left[\frac{\rho^{2}}{4\left(\sqrt{\varepsilon_{n}+1 / 4}-n-1 / 2\right)^{2}}\right. \\
& \left.+\left(\sqrt{\varepsilon_{n}+\frac{1}{4}}-n-\frac{1}{2}\right)^{2}\right]-\omega\left(c_{0}-\frac{c_{1}}{2 \sqrt{q}}+\frac{c_{2}}{2 q}\right) .
\end{aligned}
$$

By comparing (32) and (50) and using (34), we obtain $f_{n}(z)$ as

$$
\begin{aligned}
f_{n}(z)= & (-1)^{n} C^{\prime}(1)^{n}(2 \delta+1)_{n} \\
& \cdot{ }_{2} F_{1}(-n, 2 \delta+2 \gamma+n+1,2 \delta+1, z) .
\end{aligned}
$$

From (59), we determine unnormalized radial wave function with $D=5$ for various $n$ as shown in Table 6 , here $C^{\prime}$ is normalization constant.

4.2. Solution of Angular Part. In this study, the four angular parts of Dirac equations are presented in (20)-(23), so we have to solve each equation of angular Dirac equation using AIM.

4.2.1. Equation of Angular Part for $\theta_{1}$. We can solve (20) by changing it into the form of the second-order hypergeometric-type differential equation that is similar to (22) after we insert (15) in (20),

$$
\begin{aligned}
& \frac{\partial^{2} P_{1}\left(\theta_{1}\right)}{\partial \theta_{1}^{2}}-\{M+E\} \\
& \quad \cdot\left\{\frac{b_{1}^{2}+a_{1}\left(a_{1}-1\right)}{\sin _{q}^{2} \theta_{1}}+\frac{2 b_{1}\left(a_{1}-1 / 2\right) \cos _{q} \theta_{1}}{\sin _{q}^{2} \theta_{1}}\right\} \\
& \quad \cdot P_{1}\left(\theta_{1}\right)+\lambda_{1} P_{1}\left(\theta_{1}\right)=0,
\end{aligned}
$$

and substitute $\cos _{q} \theta_{1}=\sqrt{q}\left(1-2 z_{1}\right)$ into (60) so we get

$$
\begin{aligned}
& z_{1}\left(1-z_{1}\right) \frac{d^{2} P_{1}\left(z_{1}\right)}{d z_{1}{ }^{2}}+\frac{1}{2}\left(1-2 z_{1}\right) \frac{d P_{1}\left(z_{1}\right)}{d z_{1}}+\left\{\lambda_{1}\right. \\
& -\frac{\{M+E\}\left[\left(b_{1}{ }^{2}+a_{1}\left(a_{1}-1\right)\right) / q+\left(2 b_{1}\left(a_{1}-1 / 2\right) \sqrt{q}\right) / q\right]}{4 z_{1}} \\
& \left.-\frac{\{M+E\}\left[\left(b_{1}{ }^{2}+a_{1}\left(a_{1}-1\right)\right) / q-\left(2 b_{1}\left(a_{1}-1 / 2\right) \sqrt{q}\right) / q\right]}{4\left(1-z_{1}\right)}\right\} \\
& \cdot P_{1}\left(z_{1}\right)=0 .
\end{aligned}
$$

Equation (62) has two regular singular points for $z_{1}=0$ and $z_{1}=1$, and then the solution of $P_{1}\left(z_{1}\right)$ is set as

$$
P_{1}\left(z_{1}\right)=z_{1}^{\delta_{s 1}}\left(1-z_{1}\right)^{\gamma_{s 1}} f_{n_{l}}\left(z_{1}\right)
$$


TABLE 1: Relativistic energies with variation of $q$ and $n_{l}$.

\begin{tabular}{|c|c|c|c|c|c|c|c|c|c|}
\hline \multicolumn{10}{|c|}{$D=5, V_{0}=6, V_{1}=-1, a_{1}=a_{2}=a_{3}=a_{4}=b_{1}=b_{2}=b_{3}=b_{4}=2$} \\
\hline$q$ & $r_{e}(\mathrm{fm})$ & $n$ & $n_{l 1}=n_{l 2}=n_{l 3}=n_{l 4}$ & $E_{q}\left(\mathrm{fm}^{-1}\right)$ & $n_{l 1}=n_{l 2}=n_{l 3}=n_{l 4}$ & $n$ & $q$ & $r_{e}(\mathrm{fm})$ & $E_{n_{l}}\left(\mathrm{fm}^{-1}\right)$ \\
\hline 0.2 & 0.0333 & 1 & 1 & -5.2311 & 0 & 1 & 1 & 0.1671 & -5.0977 \\
\hline 0.4 & 0.0667 & 1 & 1 & -5.4766 & 1 & 1 & 1 & 0.1671 & -6.4721 \\
\hline 0.6 & 0.1001 & 1 & 1 & -5.7371 & 2 & 1 & 1 & 0.1671 & -9.1544 \\
\hline 0.8 & 0.1335 & 1 & 1 & -6.0240 & 3 & 1 & 1 & 0.1671 & -13.1643 \\
\hline 1 & 0.1671 & 1 & 1 & -6.4721 & 4 & 1 & 1 & 0.1671 & -18.5283 \\
\hline 1.2 & 0.2007 & 1 & 1 & -6.9767 & 5 & 1 & 1 & 0.1671 & -25.2837 \\
\hline
\end{tabular}

If we replace $P_{1}\left(z_{1}\right)$ in (61) with (62) and simplify it by using appropriate variable substitution as follows:

$$
\begin{aligned}
& \mathrm{O}_{s 1}{ }^{2}=\lambda_{1} \text {, } \\
& 2 \delta_{s 1}\left(2 \delta_{s 1}-1\right)=(M+E) \\
& \cdot\left(\frac{b_{1}{ }^{2}+a_{1}\left(a_{1}-1\right)}{q}-\frac{2 b_{1}\left(a_{1}-1 / 2\right) \sqrt{q}}{q}\right) \text {, } \\
& 2 \gamma_{s 1}\left(2 \gamma_{s 1}-1\right)=(M+E) \\
& \cdot\left(\frac{b_{1}^{2}+a_{1}\left(a_{1}-1\right)}{q}+\frac{2 b_{1}\left(a_{1}-1 / 2\right) \sqrt{q}}{q}\right)
\end{aligned}
$$

then (61) reduces to

$$
\begin{aligned}
& f_{n_{l}}^{\prime \prime}\left(z_{1}\right) \\
& =\frac{\left\{\left(2 \delta_{s 1}+2 \gamma_{s 1}+1\right) z_{1}-\left(2 \delta_{s 1}+1 / 2\right)\right\}}{z_{1}\left(1-z_{1}\right)} f_{n_{l}}^{\prime}\left(z_{1}\right) \\
& \quad+\frac{\left\{\left(\delta_{s 1}+\gamma_{s 1}\right)^{2}-O_{s 1}^{2}\right\}}{z_{1}\left(1-z_{1}\right)} f_{n_{l}}\left(z_{1}\right) .
\end{aligned}
$$

From (64), we get

$$
\begin{aligned}
& \lambda_{0}\left(z_{1}\right)=\frac{\left\{\left(2 \delta_{s 1}+2 \gamma_{s 1}+1\right) z_{1}-\left(2 \delta_{s 1}+1 / 2\right)\right\}}{z_{1}\left(1-z_{1}\right)} ; \\
& S_{0}\left(z_{1}\right)=\frac{\left\{\left(\delta_{s 1}+\gamma_{s 1}\right)^{2}-O_{s 1}^{2}\right\}}{z_{1}\left(1-z_{1}\right)}, \\
& \lambda_{1}\left(z_{1}\right)=\left\{\frac{\left(2 \delta_{s 1}+1 / 2\right)}{z_{1}^{2}}+\frac{\left(2 \gamma_{s 1}+1 / 2\right)}{\left(1-z_{1}\right)^{2}}\right\} \\
& +\left\{\frac{I_{s 1}}{z_{1}}+\frac{I_{s 1}}{\left(1-z_{1}\right)}\right\} \\
& +\left\{-\frac{\left(2 \delta_{s 1}+1 / 2\right)}{z_{1}}+\frac{\left(2 \gamma_{s 1}+1 / 2\right)}{\left(1-z_{1}\right)}\right\}^{2}, \\
& S_{1}\left(z_{1}\right)=\left\{-\frac{\left.I_{s 1}+\frac{I_{s 1}}{z_{1}^{2}}\right\}+\left\{\frac{I_{s 1}}{z_{1}}+\frac{I_{s 1}}{\left(1-z_{1}\right)}\right\}}{\left.\left.z_{1}\right)^{2}\right\}}\right. \\
& \quad\left\{-\frac{\left(2 \delta_{s 1}+1 / 2\right)}{z_{1}}+\frac{\left(2 \gamma_{s 1}+1 / 2\right)}{\left(1-z_{1}\right)}\right\} .
\end{aligned}
$$

TABLE 2: Relativistic energies with variation of radial quantum number $n$

\begin{tabular}{cccc}
\hline \multicolumn{5}{c}{$D=5, V_{0}=6, V_{1}=-1, \alpha=0,5\left(\mathrm{fm}^{-1}\right)$, and $r_{e}=0.1671(\mathrm{fm})$} \\
$n$ & $a_{1}=a_{2}=a_{3}=a_{4}=b_{1}=b_{2}=b_{3}=b_{4}=2$ \\
\hline 0 & $q$ & $n_{l 1}=n_{l 2}=n_{l 3}=n_{l 4}$ & $E_{n}\left(\mathrm{fm}^{-1}\right)$ \\
1 & 1 & 1 & -6.4725 \\
2 & 1 & 1 & -6.4721 \\
3 & 1 & 1 & -6.4717 \\
4 & 1 & 1 & -6.4713 \\
\hline & 1 & 1 & -6.4709 \\
\hline
\end{tabular}

TABLE 3: The relativistic energies with variation of potential parameters $a_{1} ; a_{2} ; a_{3} ; a_{4}$ and $b_{1} ; b_{2} ; b_{3} ; b_{4}$ with $M=5 ; C_{s}=0 ; n_{r}=0$; $n_{l 1}=n_{l 2}=n_{l 3}=n_{l 4}=0 ; V_{0}=6 ; \alpha=0.5 ; q=1 ; V_{1}=-1$; and $r_{e}=0.1671$.

\begin{tabular}{ccccccccc}
\hline$a_{1}$ & $b_{1}$ & $a_{2}$ & $b_{2}$ & $a_{3}$ & $b_{3}$ & $a_{4}$ & $b_{4}$ & $E_{a b}(\mathrm{fm})$ \\
\hline 2 & 2 & 2 & 2 & 2 & 2 & 2 & 2 & -5.0980 \\
4 & 4 & 2 & 2 & 2 & 2 & 2 & 2 & -5.0821 \\
6 & 6 & 2 & 2 & 2 & 2 & 2 & 2 & -5.0561 \\
8 & 8 & 2 & 2 & 2 & 2 & 2 & 2 & -5.0382 \\
10 & 10 & 2 & 2 & 2 & 2 & 2 & 2 & -5.0269 \\
2 & 2 & 4 & 4 & 2 & 2 & 2 & 2 & -5.0528 \\
2 & 2 & 6 & 6 & 2 & 2 & 2 & 2 & -5.0319 \\
2 & 2 & 8 & 8 & 2 & 2 & 2 & 2 & -5.0211 \\
2 & 2 & 10 & 10 & 2 & 2 & 2 & 2 & -5.0171 \\
2 & 2 & 2 & 2 & 4 & 4 & 2 & 2 & -5.0383 \\
2 & 2 & 2 & 2 & 6 & 6 & 2 & 2 & -5.0136 \\
2 & 2 & 2 & 2 & 8 & 8 & 2 & 2 & -5.0084 \\
2 & 2 & 2 & 2 & 10 & 10 & 2 & 2 & -5.0055 \\
2 & 2 & 2 & 2 & 2 & 2 & 4 & 4 & -5.0453 \\
2 & 2 & 2 & 2 & 2 & 2 & 6 & 6 & -5.0266 \\
2 & 2 & 2 & 2 & 2 & 2 & 8 & 8 & -5.0200 \\
2 & 2 & 2 & 2 & 2 & 2 & 10 & 10 & -5.0156 \\
\hline
\end{tabular}

By using (65)-(68) and using relation of (31), we get

$$
\left(\ell_{1}^{\prime 2}\right)_{n_{l}}=\left(\delta_{s 1}+\gamma_{s 1}+n_{l}\right)^{2}
$$


where $n_{l}$ is orbital quantum number. The angular wave function of (64) is determined by using (34) and then we get

$$
\begin{aligned}
f_{n_{l}}= & (-1)^{n_{l}} C^{\prime}(1)^{n_{l}}\left(2 \delta_{s 1}+\frac{1}{2}\right)_{n_{l}} \\
& \cdot{ }_{2} F_{1}\left(-n_{l}, 2 \delta_{s 1}+2 \gamma_{s 1}+n_{l}, 2 \delta_{s 1}+\frac{1}{2}, z\right),
\end{aligned}
$$

where $C^{\prime}$ is normalization constant and ${ }_{2} F_{1}(a, b ; c ; z)$ is hypergeometry function. From (70) we solve unnormalized angular wave function as function of $z$ completely for variation of $n_{l}$ as shown in Table 7.

4.2.2. Equation of Angular Part for $\theta_{2}, \theta_{3}$, and $\theta_{4}$. Solutions for $\theta_{2}, \theta_{3}$, and $\theta_{4}$ are determined by the same way as the solution for $\theta_{1}$, by setting subscript $i$ with 2,3 , and 4 . So we get the solution for orbital quantum number for $\theta_{2}, \theta_{3}$, and $\theta_{4}$, respectively, as follows:

$$
\begin{aligned}
& \left(\ell_{2}^{\prime}\right)_{n_{l}}=\delta_{s 2}+\gamma_{s 2}+\left(n_{l}-\frac{1}{2}\right), \text { for } \theta_{2} n_{l}=0,1, \ldots, \\
& \left(\ell_{3}^{\prime}\right)_{n_{l}}=\delta_{s 3}+\gamma_{s 3}+\left(n_{l}-1\right), \text { for } \theta_{3} n_{l}=0,1, \ldots, \\
& \left(\ell_{4}^{\prime}\right)_{n_{l}}=\delta_{s 4}+\gamma_{s 4}+\left(n_{l}-\frac{3}{2}\right), \text { for } \theta_{4} n_{l}=0,1, \ldots,
\end{aligned}
$$

where

$$
\begin{aligned}
& \delta_{s 2}=\frac{\sqrt{\lambda_{1}+(M+E)\left(\left(b_{2}^{2}+a_{2}\left(a_{2}-1\right)\right) / q-2 b_{2}\left(a_{2}-1 / 2\right) / \sqrt{q}\right)}+1 / 2}{2}, \\
& \delta_{s 3}=\frac{\sqrt{\lambda_{2}+1 / 4+(M+E)\left(\left(b_{3}^{2}+a_{3}\left(a_{3}-1\right)\right) / q-2 b_{3}\left(a_{3}-1 / 2\right) / \sqrt{q}\right)}+1 / 2}{2}, \\
& \delta_{s 4}=\frac{\sqrt{\lambda_{3}-1 / 2+(M+E)\left(\left(b_{4}^{2}+a_{4}\left(a_{4}-1\right)\right) / q-2 b_{4}\left(a_{4}-1 / 2\right) / \sqrt{q}\right)}+1 / 2}{2}, \\
& \gamma_{s 2}=\frac{\sqrt{\lambda_{1}+(M+E)\left(\left(b_{2}^{2}+a_{2}\left(a_{2}-1\right)\right) / q+2 b_{2}\left(a_{2}-1 / 2\right) / \sqrt{q}\right)+1 / 2}}{2} \\
& \gamma_{s 3}=\frac{\sqrt{\lambda_{2}+(M+E)\left(\left(b_{3}^{2}+a_{3}\left(a_{3}-1\right)\right) / q+2 b_{3}\left(a_{3}-1 / 2\right) / \sqrt{q}\right)+1 / 4}+1 / 2}{2}, \\
& \gamma_{s 4}=\frac{\sqrt{\lambda_{3}-1 / 2+(M+E)\left(\left(b_{4}^{2}+a_{4}\left(a_{4}-1\right)\right) / q+2 b_{4}\left(a_{4}-1 / 2\right) / \sqrt{q}\right)}+1 / 2}{2} .
\end{aligned}
$$

The unnormalized angular wave functions for $\theta_{2}, \theta_{3}$, and $\theta_{4}$ are in the same pattern as the angular wave function for $\theta_{1}$ so we obtain the last three angular wave functions by simple change of parameters in (70) with parameters in (74)-(79).

The relativistic energy levels are calculated numerically using Matlab program R2013a. Table 1 shows the relativistic energy levels as a function of deformation parameter $q$; the relativistic energy $E$ decreases when deformation parameter $q$ increases. Here we apply the value of $q$ from 0.2 until 1.2 with step 0.2 . The relativistic energy levels as a function of orbital quantum numbers $n_{l}$ are also shown in Table 1. The magnitude of energies $E$ decreases when the orbital quantum numbers $n_{l}$ increase. The relativistic energy levels as a function of radial quantum number $n$ are shown in Table 2. The relativistic energies $E$ increase when radial quantum number $n$ increases. The relativistic energies numerically also change as a function of potential parameters $a_{i}$ and $b_{i}$ where $i=1,2,3,4$ are the components of $i$ th noncentral potential (Table 3 ). The relativistic energies $E$ increase when both potential parameters in each potential component increase. This suggests that the bounded energies become less bounded with increasing of potential parameters. The unnormalized angular wave functions are listed in Table 4. The unnormalized radial wave functions are plotted by using (46) and (59) as shown in Figure 1. From Figures 1(a)-1(c), it is seen that the amplitude of the wave function increases when the orbital quantum number increases. This suggests that the probability of finding particles is larger for higher orbital quantum number $n_{l}$.

\section{Special Case for Three-Dimensional System}

In three-dimensional case, $D=3$, the bound state relativistic energy levels are calculated numerically from (58), (43), (44), (56), (69), (74), and (77) using Matlab software R2013a and are presented in Tables 5, 6, and 7. By using (44) and (62), the unnormalized radial wave functions are listed in Table 8. In Table 5, the relativistic energy levels decrease 
TABLE 4: The unnormalized upper angular wave function for higher dimension $D=5$.

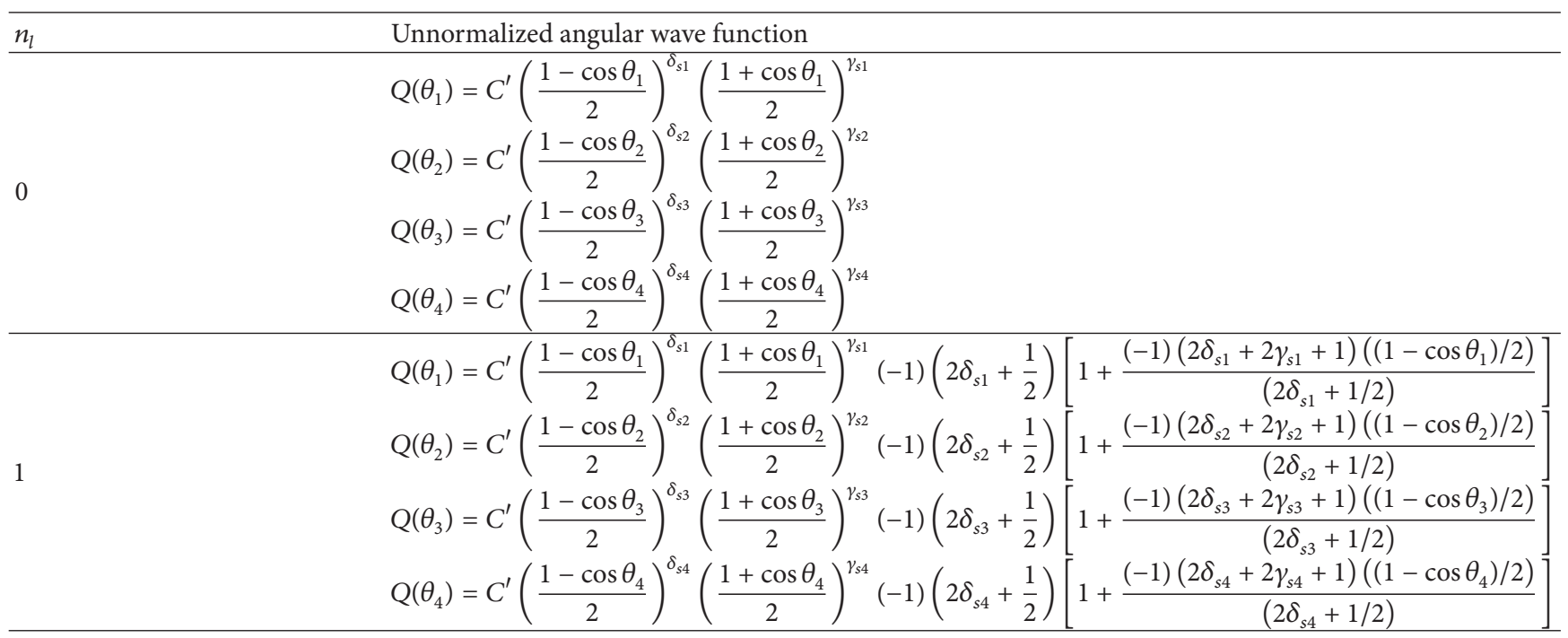

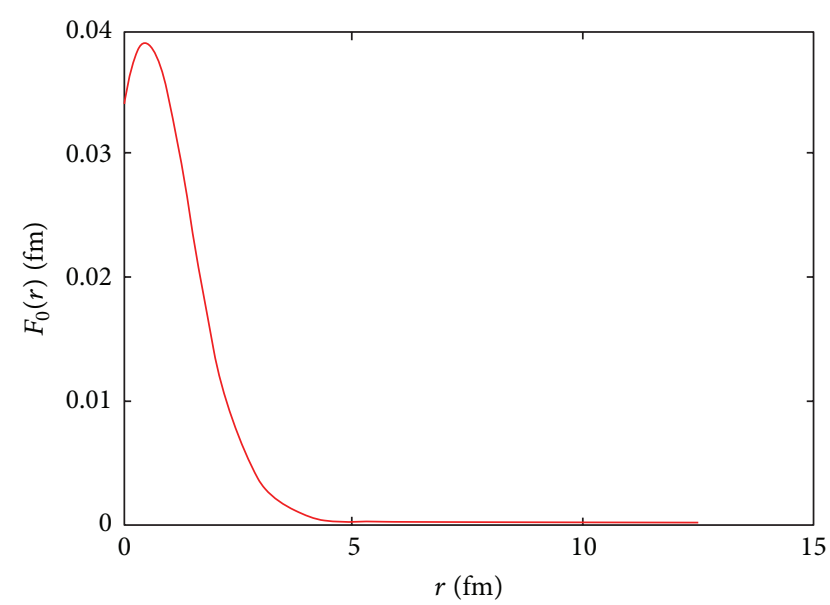

(a)

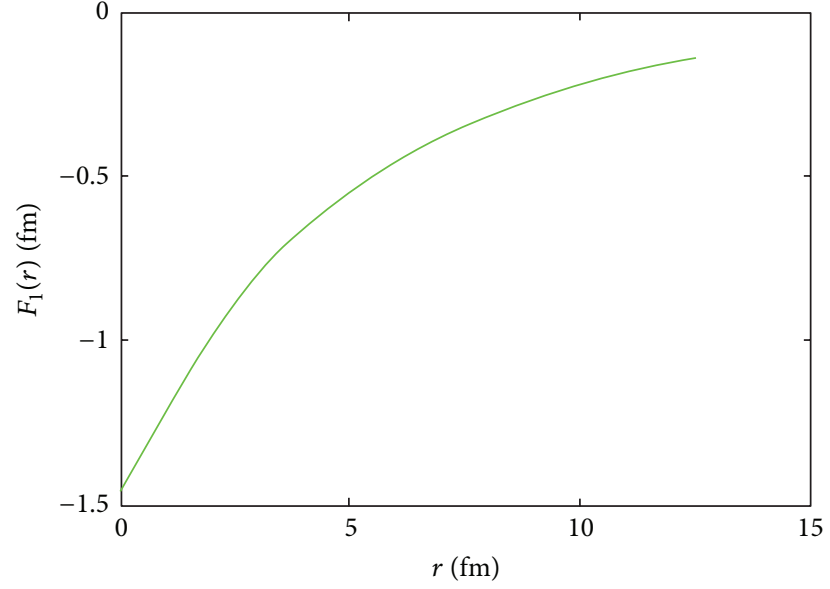

(b)

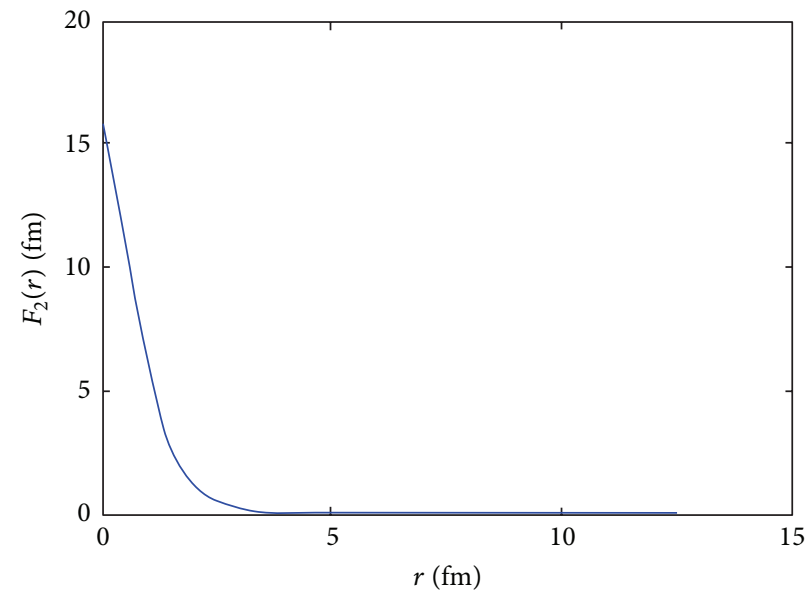

(c)

FIGURE 1: Unnormalized radial wave functions versus hyperspherical radius $r$ with variation of radial quantum number, where $M=5 \mathrm{fm}^{-1}$, $C_{s}=0, V_{0}=6, V_{1}=-1, r_{e}=0.1671 \mathrm{fm}$, and $n_{l i}=1, i=1,2,3,4$. (a) For $n=0$, (b) for $n=1$, and (c) for $n=2$. 
TABLE 5: Relativistic energy with variation of $q$ and $n_{l}$.

\begin{tabular}{|c|c|c|c|c|c|c|c|c|c|}
\hline \multirow[b]{2}{*}{$q$} & \multirow[b]{2}{*}{$r_{e}(\mathrm{fm})$} & \multicolumn{7}{|c|}{$V_{0}=6, V_{1}=-1, \alpha=0,5\left(\mathrm{fm}^{-1}\right) a_{1}=a_{2}=b_{1}=b_{2}=2$} & \multirow[b]{2}{*}{$E_{n_{l}}\left(\mathrm{fm}^{-1}\right)$} \\
\hline & & $n$ & $n_{l 1}=n_{l 2}$ & $E_{q}\left(\mathrm{fm}^{-1}\right)$ & $n_{l 1}=n_{l 2}$ & $n_{r}$ & $q$ & $r_{e}(\mathrm{fm})$ & \\
\hline 0.2 & 0.0333 & 1 & 1 & -5.1360 & 0 & 1 & 1 & 0.1671 & -5.1394 \\
\hline 0.4 & 0.0667 & 1 & 1 & -5.2867 & 1 & 1 & 1 & 0.1671 & -6.1034 \\
\hline 0.6 & 0.1001 & 1 & 1 & -5.4552 & 2 & 1 & 1 & 0.1671 & -7.9374 \\
\hline 0.8 & 0.1335 & 1 & 1 & -5.6616 & 3 & 1 & 1 & 0.1671 & -10.6614 \\
\hline 1 & 0.1671 & 1 & 1 & -6.1034 & 4 & 1 & 1 & 0.1671 & -14.3053 \\
\hline 1.2 & 0.2007 & 1 & 1 & -6.5579 & & & & & \\
\hline
\end{tabular}

TABLE 6: Relativistic energies with variation of $n_{r}$ and potential parameter.

\begin{tabular}{|c|c|c|c|c|c|c|c|}
\hline \multirow[b]{2}{*}{$n$} & \multicolumn{7}{|c|}{$M=5, V_{0}=6, V_{1}=-1, q=1, \alpha=0,5\left(\mathrm{fm}^{-1}\right)$, and $r_{e}=0.1671(\mathrm{fm})$} \\
\hline & $n_{l 1}=n_{l 2}$ & $a_{1}=a_{2}=b_{1}=b_{2}$ & $E_{n n_{l 1} n_{l 2}}\left(\mathrm{fm}^{-1}\right)$ & $n$ & $n_{l 1}=n_{l 2}$ & $a_{1}=a_{2}=b_{1}=b_{2}$ & $E_{n n_{l 1} n_{l 2}}\left(\mathrm{fm}^{-1}\right)$ \\
\hline 0 & 1 & 2 & -6.1042 & 0 & 1 & 0 & -0.0616 \\
\hline 1 & 1 & 2 & -6.1034 & 1 & 1 & 0 & -0.0564 \\
\hline 2 & 1 & 2 & -6.1024 & 2 & 1 & 0 & -0.0509 \\
\hline 3 & 1 & 2 & -6.1014 & 3 & 1 & 0 & -0.0454 \\
\hline 4 & 1 & 2 & -6.1004 & 4 & 1 & 0 & -0.0398 \\
\hline
\end{tabular}

TABLE 7: Relativistic energies for $M=1 \mathrm{fm}^{-1}, C_{s}=0, V_{0}=6$, and $V_{1}=-1$ without presence of $q$-deformed noncentral potentials.

\begin{tabular}{ccccccc}
\hline$\ell$ & $n$ & $K$ & $(\ell, j)$ & $E_{n K}^{s s}\left(\mathrm{fm}^{-1}\right), q=1$, & $E_{n K}^{s s}\left(\mathrm{fm}^{-1}\right), q=1.2$, & $r_{e}=0.2007$ \\
\hline 1 & 0 & -2 & $0 \mathrm{p}_{3 / 2}$ & -0.5028 & -0.5014 & -0.5006 \\
2 & 0 & -3 & $0 \mathrm{~d}_{5 / 2}$ & -0.5008 & -0.5002 & -0.4999 \\
3 & 0 & -4 & $0 \mathrm{f}_{7 / 2}$ & -0.5002 & -0.4999 & -0.4996 \\
4 & 0 & -5 & $0 \mathrm{~g}_{9 / 2}$ & -0.5000 & -0.4998 & -0.4995 \\
1 & 1 & -2 & $1 \mathrm{f}_{3 / 2}$ & -0.5042 & -0.5028 & -0.5020 \\
2 & 1 & -3 & $1 \mathrm{~d}_{5 / 2}$ & -0.5013 & -0.5007 & -0.4999 \\
3 & 1 & -4 & $1 \mathrm{f}_{7 / 2}$ & -0.5005 & -0.5002 & -0.4997 \\
4 & 1 & -5 & $1 g_{9 / 2}$ & -0.5002 & -0.4999 & -0.5006 \\
1 & 0 & 1 & $0 \mathrm{p}_{1 / 2}$ & -0.5028 & -0.5014 & -0.4999 \\
2 & 0 & 2 & $0 \mathrm{~d}_{3 / 2}$ & -0.5008 & -0.5002 & -0.4996 \\
3 & 0 & 3 & $0 \mathrm{f}_{5 / 2}$ & -0.5002 & -0.4999 & -0.4995 \\
4 & 0 & 4 & $0 g_{7 / 2}$ & -0.5000 & -0.4998 & -0.5020 \\
1 & 1 & 1 & $\operatorname{lp}_{1 / 2}$ & -0.5042 & -0.5028 & -0.5003 \\
2 & 1 & 2 & $1 \mathrm{~d}_{3 / 2}$ & -0.5013 & -0.5007 & -0.4999 \\
3 & 1 & 3 & $1 f_{5 / 2}$ & -0.5005 & -0.5002 & -0.4997 \\
4 & 1 & 4 & $1 g_{7 / 2}$ & -0.5002 & -0.4999 & \\
\hline
\end{tabular}

TABLE 8: Unnormalized upper radial wave function for dimension $D=3$.

\begin{tabular}{ll}
\hline$n$ & Radial wave function \\
\hline 0 & $F_{0}(r)=C^{\prime}\left(\frac{1-\tanh _{q} \alpha r}{2}\right)^{\delta}\left(\frac{1+\tanh _{q} \alpha r}{2}\right)^{\gamma}$ \\
\hline & $F_{1}(r)=C^{\prime}\left(\frac{1-\tanh _{q} \alpha r}{2}\right)^{\delta}\left(\frac{1+\tanh _{q} \alpha r}{2}\right)^{\gamma}(-1) C^{\prime}(2 \delta+1)\left(1+\frac{(-1)_{1}(2 \delta+2 \gamma+2)_{1}\left(\left(1-\tanh _{q} \alpha r\right) / 2\right)^{1}}{(2 \delta+1)_{1} 1 !}\right.$ \\
\hline
\end{tabular}


TABLE 9: The unnormalized upper angular wave function for dimension $D=3$.

\begin{tabular}{ll}
\hline$n_{l}$ & Unnormalized angular wave function \\
\hline 0 & $Q\left(\theta_{1}\right)=C^{\prime}\left(\frac{1-\cos \theta_{1}}{2}\right)^{\delta_{s 1}}\left(\frac{1+\cos \theta_{1}}{2}\right)^{\gamma_{s 1}}$ \\
\hline & $Q\left(\theta_{2}\right)=C^{\prime}\left(\frac{1-\cos \theta_{2}}{2}\right)^{\delta_{s 2}}\left(\frac{1+\cos \theta_{2}}{2}\right)^{\gamma_{s 2}}$ \\
\hline & $Q\left(\theta_{1}\right)=C^{\prime}\left(\frac{1-\cos \theta_{1}}{2}\right)^{\delta_{s 1}}\left(\frac{1+\cos \theta_{1}}{2}\right)^{\gamma_{s 1}}(-1)\left(2 \delta_{s 1}+\frac{1}{2}\right)\left[1+\frac{(-1)\left(2 \delta_{s 1}+2 \gamma_{s 1}+1\right)\left(\left(1-\cos \theta_{1}\right) / 2\right)}{\left(2 \delta_{s 1}+1 / 2\right)}\right.$ \\
& $Q\left(\theta_{2}\right)=C^{\prime}\left(\frac{1-\cos \theta_{2}}{2}\right)^{\delta_{s 2}}\left(\frac{1+\cos \theta_{2}}{2}\right)^{\gamma_{s 2}}(-1)\left(2 \delta_{s 2}+\frac{1}{2}\right)\left[1+\frac{(-1)\left(2 \delta_{s 2}+2 \gamma_{s 2}+1\right)\left(\left(1-\cos \theta_{2}\right) / 2\right)}{\left(2 \delta_{s 2}+1 / 2\right)}\right.$ \\
\hline
\end{tabular}

with the increase of both deformation parameter $q$ and orbital quantum number $n_{l}$, where $n_{l 1}$ is orbital quantum number for $\theta_{1}$ and $n_{l 2}$ is orbital quantum number for $\theta_{2}$. From Table 7, we can conclude that the value of relativistic energies increases with the increase of the radial quantum number $n$ and with either the presence or absence of angular potential parameter. The angular potential parameters $a$ and $b$ influence the relativistic energy level, where the relativistic energy levels increase by increasing the angular potential parameter. The unnormalized upper radial wave functions are listed in Table 8 when the radial quantum numbers are $n=0$ and $n=1$ and $C^{\prime}$ is the normalization constant. The unnormalized angular wave functions as functions of $\theta_{1}$ and $\theta_{2}$ are listed in Table 9. Without the presence of the noncentral potential, degeneracy energy spectra occur in the spin doublets, with quantum numbers $(n, \ell, j=\ell+1 / 2)$ and $(n, \ell, j=\ell-1 / 2)$, where $n, \ell$, and $j$ are the radial, the orbital, and the total angular momentum quantum numbers, respectively, for example, $\left(n \mathrm{p}_{1 / 2}, n \mathrm{p}_{3 / 2}\right)$ for $\ell=1$ but for difference values of $j=1 / 2$ and $3 / 2,\left(n \mathrm{~d}_{3 / 2}, n \mathrm{~d}_{5 / 2}\right)$ for $\ell=2$ with $\left(j=3 / 2\right.$ and 5/2), $\left(n f_{5 / 2}, n f_{7 / 2}\right)$ for $\ell=3$ with $(j=5 / 2$ and $7 / 2)$, and $\left(n g_{7 / 2}, n g_{9 / 2}\right)$ for $\ell=4$ with $(j=7 / 2$ and $9 / 2)$. The degeneracy energies can be removed with the presence of noncentral potential, by changing $\ell \rightarrow K$, where $K=-\ell-1$ and $K=\ell$ for $K<0$ and $K>0$.

\section{Conclusion}

In this study, we have obtained the bound state solution of the 5-dimensional Dirac equation with separable $q$-deformed quantum potential under condition of spin symmetry. The upper component of Dirac spinors and relativistic energy have been obtained using Asymptotic Iteration Method (AIM). The numerical result shows that the bound state relativistic energy level for spin symmetry case with both dimensions $D=5$ and $D=3$ increases with increasing of radial quantum number $n$ and decreases with increasing of both deformation parameter $q$ and orbital quantum number $n_{l}$. The degeneracy energy states occur in the absence of $q$ deformed noncentral potential.

\section{Competing Interests}

The authors declare that they have no competing interests.

\section{Acknowledgments}

This Research is partly supported by Higher Education Project Grant with contract no. 632/UN27/27.21/LT/2016.

\section{References}

[1] W. Greiner, Relativistic Quantum Mechanics, Springer, New York, NY, USA, 3rd edition, 2000.

[2] B. J. Falaye and K. J. Oyewumi, "Solutions of the Dirac equation with spin and pseudospin symmetry for the trigonometric Scarf potential in D-dimensions," The African Review of Physics, vol. 6, article 0025, 2011.

[3] S. M. Ikhdair, "Approximate solutions of the Dirac equation for the Rosen-Morse potential including the spin-orbit centrifugal term," Journal of Mathematical Physics, vol. 51, no. 2, Article ID 023525, 2010.

[4] J. N. Ginocchio, "Pseudospin as a relativistic symmetry," Physical Review Letters, vol. 78, no. 3, pp. 436-439, 1997.

[5] R. A. Sari, A. Suparmi, and C. Cari, "Solution of Dirac equation for Eckart potential and trigonometric Manning Rosen potential using asymptotic iteration method," Chinese Physics B, vol. 25, no. 1, Article ID 010301, 2015.

[6] A. Kurniawan, A. Suparmi, and C. Cari, "Approximate analytical solution of the Dirac equation with q-deformed hyperbolic Pöschl-Teller potential and trigonometric Scarf II non-central potential," Chinese Physics B, vol. 24, no. 3, Article ID 030302, 2015.

[7] A. Suparmi, C. Cari, U. A. Deta, A. S. Husein, and H. Yuliani, "Exact solution of Dirac equation for q-deformed trigonometric Scarf potential with q-deformed trigonometric tensor coupling potential for spin and pseudospin symmetries using Romanovski polynomial," Journal of Physics: Conference Series, vol. 539, no. 1, Article ID 012004, 2014.

[8] M. Hamzavi and A. A. Rajabi, "Generalized nuclear WoodsSaxon potential under relativistic spin symmetry limit," ISRN High Energy Physics, vol. 2013, Article ID 987632, 7 pages, 2013.

[9] M. Sameer, "Approximate relativistic bound states of a particle in Yukawa field with Coulomb tensor interaction," Physica Scripta, vol. 87, no. 3, Article ID 035002, 2013.

[10] Suparmi and Cari, "Bound state solution of Dirac equation for generalized Pöschl-Teller plus trigomometric Pöschl-Teller non-central potential using SUSY quantum mechanics," Journal of Mathematical and Fundamental Sciences, vol. 46, no. 3, pp. 205-223, 2014. 
[11] O. Bayrak and I. Boztosun, "The pseudospin symmetric solution of the Morse potential for any $\kappa$ state," Journal of Physics A, vol. 40, no. 36, pp. 11119-11127, 2007.

[12] H. Hassanabadi, E. Maghsoodi, S. Zarrinkamar, and H. Rahimov, "Dirac equation under scalar, vector, and tensor cornell interactions," Advances in High Energy Physics, vol. 2012, Article ID 707041, 17 pages, 2012.

[13] H. Hassanabadi, E. Maghsoodi, A. N. Ikot, and S. Zarrinkamar, "Dirac equation under scalar and vector generalized isotonic oscillators and cornell tensor interaction," Advances in High Energy Physics, vol. 2014, Article ID 831938, 7 pages, 2014.

[14] A. Lopez-Ortega, “The Dirac equation in D-dimensional spherically symmetric spacetimes," https://arxiv.org/abs/0906.2754.

[15] R. L. Hall and P. Zorin, "Refined comparison theorems for the Dirac equation in $d$ dimensions," Annalen der Physik, vol. 527, no. 5-6, pp. 408-422, 2015.

[16] A. N. Ikot, H. Hassanabadi, E. Maghsoodi, and S. Zarrinkamar, "D-dimensional Dirac equation for energy-dependent pseudoharmonic and Mie-type potentials via SUSYQM," Communications in Theoretical Physics, vol. 61, no. 4, pp. 436-446, 2014.

[17] C. Cari, A. Suparmi, M. Yunianto, and A. S. Husein, "Solution of D-dimensional Dirac equation for Coulombic potential using NU method and its thermodynamics properties," AIP Conference Proceeding, vol. 1710, Article ID 030009, 2016.

[18] A. Suparmi, C. Cari, B. N. Pratiwi, and U. A. Deta, "Solution of D dimensional Dirac equation for hyperbolic tangent potential using NU method and its application in material properties," AIP Conference Proceedings, vol. 1710, Article ID 030010, 2016.

[19] A. S. Dutra, "Mapping deformed hyperbolic potentials into nondeformed ones," Physics Letters A, vol. 339, no. 3-5, pp. 252254, 2005.

[20] T. Das, "Exact solutions of the Klein-Gordon equation for q-deformed manning-rosen potential via asymptotic iteration method," https://arxiv.org/abs/1409.1457v1.

[21] A. Soylu, O. Bayrak, and I. Boztosun, "An approximate solution of Dirac-Hulthén problem with pseudospin and spin symmetry for any $\kappa$ state," Journal of Mathematical Physics, vol. 48, no. 8, Article ID 082302, 2007.

[22] A. Soylu, O. Bayrak, and I. Boztosun, " $K$ state solutions of the Dirac equation for the Eckart potential with pseudo-spin and spin symmetry," Journal of Physics A: Mathematical and Theoretical, vol. 41, Article ID 065308, 2008.

[23] G. Kocak, O. Bayrak, and I. Boztosun, "Supersymmetric solution of Schrödinger equation by using the asymptotic iteration method," Annalen der Physik, vol. 524, no. 6-7, pp. 353-359, 2012.

[24] B.-H. Nam, J.-Y. Moon, E.-H. Park et al., "Conserved gene structure and function of interleukin-10 in teleost fish," Journal of Animal and Veterinary Advances, vol. 13, no. 12, pp. 774-782, 2014. 

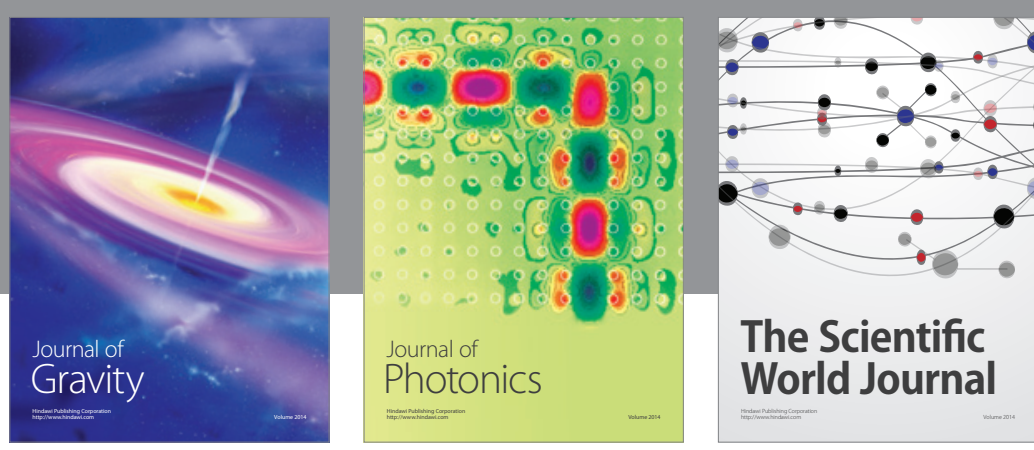

The Scientific World Journal
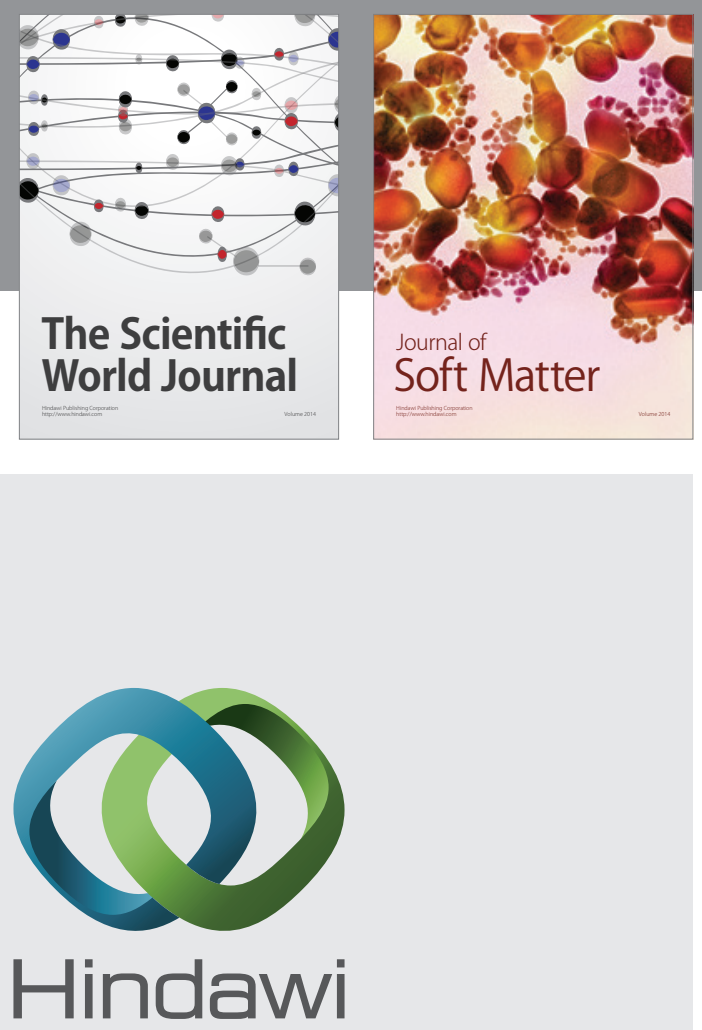

Submit your manuscripts at

http://www.hindawi.com

nternational Journal of

Statistical Mechanics
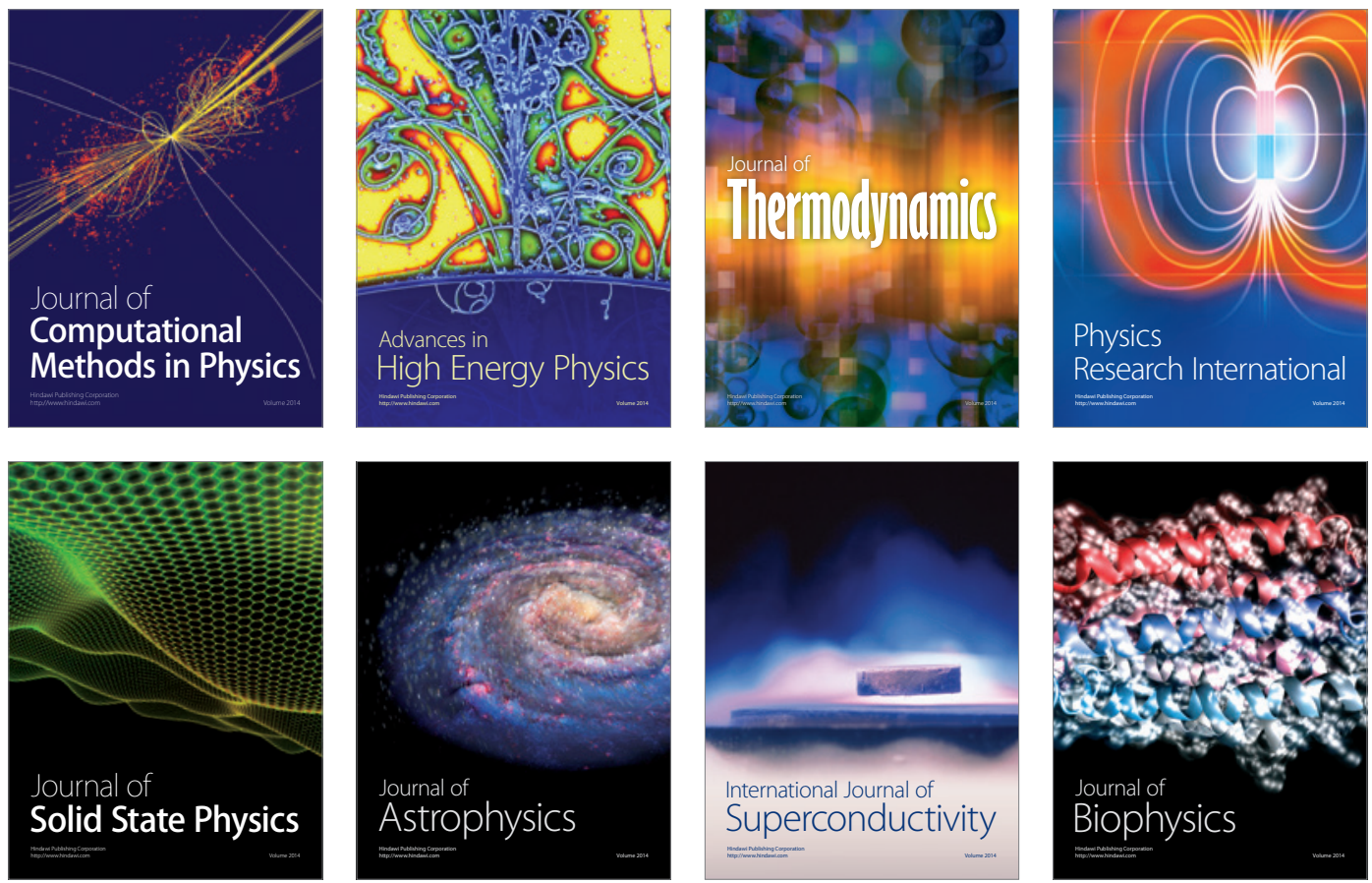
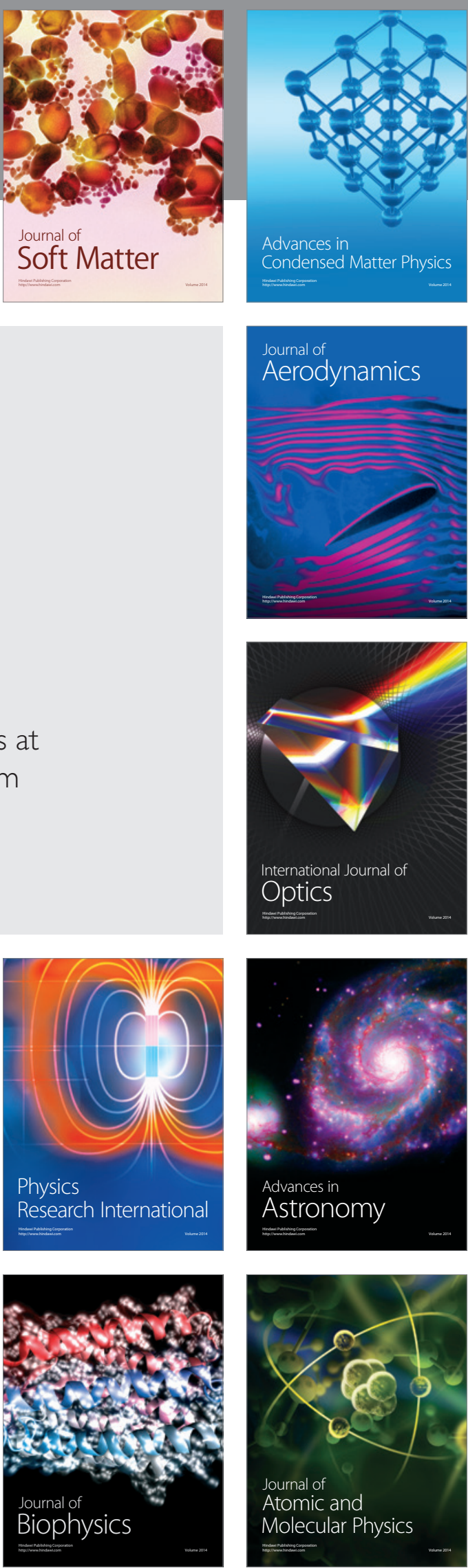